\title{
Design and Implementation on Auto Test Set of an Airborne Radar
}

\author{
Fulu Jin Yunpeng Li \\ Information Countermeasure Department \\ Aviation University of Air Force \\ ChangChun, China \\ e-mail:hbdgjfl1973@163.com
}

\author{
Hongrui Wang \\ The 1st TrainingCorps of Pilot Instructor Training Base \\ Aviation University of Air Force \\ ChangChun, China \\ e-mail:315252159@qq.com
}

\begin{abstract}
To automatic test the function and performance of an airborne radar, changeable test adapter is adopted to implement the hardware and software design of the automatic test set of the antenna, transceiver and indicator of the radar based on AT89C52. Problems such as the different types of interfaces, the various kinds of signals and the test of microwave signal are solved successfully and the objectives of resource sharing and automatic test are realized. The test software is designed by modular structure, and with the help of automatic test set hardware, the required test items of the radar system are experimented and the test process control succeeded. Experiment results show that the automatic test set performs steadily and the results meet the requirements of the airborne radar. The set has the advantages of intelligent, manageable and reducing artificial errors. It provides effective guarantees for radar's maintenance, fault diagnosis and fault detection, and has a wide application prospect with low cost.
\end{abstract}

Keywords-autotest;airborne radar;single chip microcomputer

\section{INTRODUCTION (HEADING 1)}

The airborne radar on the equipment of certain airplane consists of antenna, transceiver extension and display device. It is mainly used to detect the target of the airplane which is in the front air and on the ground, and show the relative distance and position and some other parameters of the goal to the airplane on the digital indicator so that the pilot can judge and choose the safe airline accurately. When the radar breaks down, the airplane will have to go back to the manufacturer for maintaining because there is no testing equipment in the end user. High maintenance costs and long period of repair has severely influenced the work efficiency of the airplane. So it is necessary to develop a set of testing equipment which matches the radar, so as to realize the radar maintenance and the fault detection of the above three components.

Abroad study of automatic test technology is more mature, which, however, did not provide specific technical details. In recent years, a lot of deep study has been done domestically for radar testing techniques and its automatic test equipment, which lays a solid foundation for the development of the automatic test bench ${ }^{[1]-[3]}$. We take AT89C52 micro processor as the core to design an automatic test board for this type of air borne radar, of which the software is modular in structure ${ }^{[4]}$, and it can accomplish automatic test on function and performance of the three components of the radar. It has the advantages of high degree of intelligence, simple operation, less error on human maintenance etc., which provides effective protection for maintenance, fault detection and diagnosis in the process of using the radar.

\section{HARDWARE CIRCUIT DESIGN}

\section{A. The main control circuit}

The main control circuit is used to complete part of the logic of the automated test control and data processing, it is composed by microprocessor, keyboard , and display circuit, the D/A conversion circuit, digital I/O expansion circuit, the relay board and some others. Work principle block diagram is shown in Fig.1.

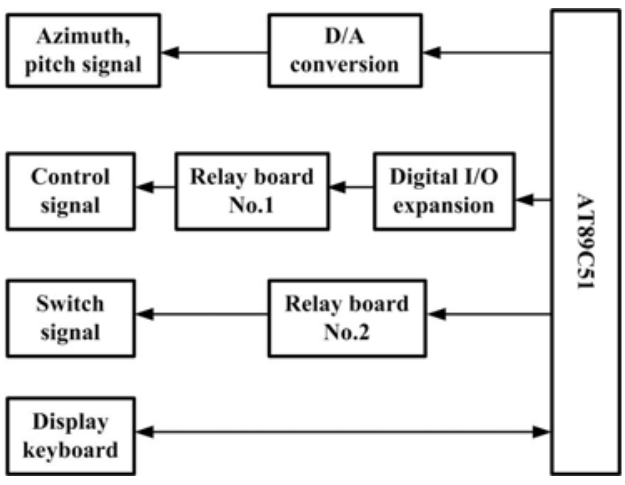

Figure 1. Schematic of test bench hardware circuit

Microprocessor uses AT89C52 produced by ATMEL Corporation, which produces all the control instructions. AT89C52 also gives different control instructions to the part of the circuit through the bus and forms the test control signal and switching signal respectively. Keyboard and display circuit consists of a $128 \times 64$-point Chinese ACM12864JHZ LCD monitor and five micro- button switch and the system adopts Chinese language with good human computer interaction interface. D/A conversion circuit completes the conversion of digital to analog and simulates the output signal of the radar antenna to add to the radar antennae extension which is tested. The digital I / O expansion circuit expands microcontroller I / O port and increases the control interface of the main control circuit which is used to control the relay board 1 to generate a test control signal mainly. Relay board 2 selects Board DAT$7675 \mathrm{~B}$ which is used in industrial site. Board DAT-7675B is 
a 24 single-pole double-throw relay board, and here it is mainly used to supply switch power when radar is tested.

\section{B. Low-frequency signal measurement circuit}

Fig. 2 shows the low-frequency signal measurement circuit. The measured signal can be directly introduced from the radar connector adapter, which will be modified in the adapter, so that the range can be within the scope that the $\mathrm{A} / \mathrm{D}$ conversion device can withstand $(0- \pm 10 \mathrm{~V})$; the selected teat signal is output to the A/D conversion circuit, converting the analog signal into digital one and eventually sending it to the control and display circuit. Measurement results will be revealed via LCD monitor.

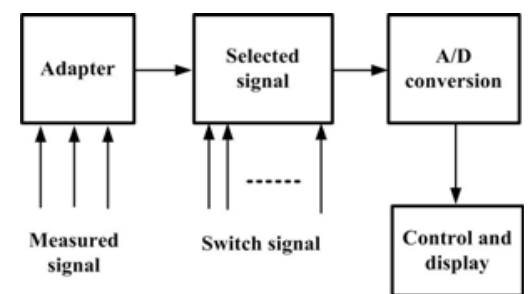

Figure 2. Schematic of low-frequency signal measurement

\section{Microwave signal measurement circuit}

The microwave tip of the radar, which is in the condition of high power, high voltage and high frequency, shares the highest failure rate among all radar components. Therefore, measurement of the microwave signal parameters of the radar is particularly important. Measurement of microwave signal mainly includes measurement of radar frequency, power and receiver sensitivity, as is shown in Schematic Fig.3. In terms of the signal that the radar transmits is of high power, the signal must be eroded to the scope that the bench can bear when measuring microwave signal. Here we utilize directional coupler $(60 \mathrm{~dB})$ to achieve high-power microwave signal attenuation. Only $1 / 10^{6}$ of the energy generated by transmitter is sent to test bench through coupling tip of the directional coupler. The rest of the energy will be absorbed by the load absorption device which is connected to the passthrough port of the directional coupler, so as to avoid the radiation into space.

\section{a) Frequency-measurement circuit}

The radar-launching signals generated by radar transceiver extension must be within the set range. Otherwise, it will influence the directional pattern of radar antennae, making its main direction deviate from the direction perpendicular to the antennae, then reducing proceeds, weakening the fight function of radar as well. Frequencymeasurement circuit is mainly constituted by sweepingfrequency signal source, mixer (converter) and low-pass filter. Sweep frequency signal source can shift in the range of $100 \mathrm{MHz}$ up and down of center frequency, $1 \mathrm{MHz}$ per step. Signals sent by signal source can make frequency mixing with radar-launching signals sent by directional coupler, then beat frequency will be sent to low-pass filter. The cut-off frequency of low pass filter is $1 \mathrm{MHz}$. That is to say, when the rate of beat frequency is less than $1 \mathrm{MHz}$, low-pass filter has the maximum output, and signal frequency sent by sweeping- frequency signal source is thought to be the frequency of radar-launching signals at this moment, the margin for error of which is not more than $1 \mathrm{MHz}$.Taking out of control voltage output from control circuit, which has a one-to-one correlation with oscillation frequency of sweeping-frequency signal source. Then the measured rate figure will be shown on the monitor.

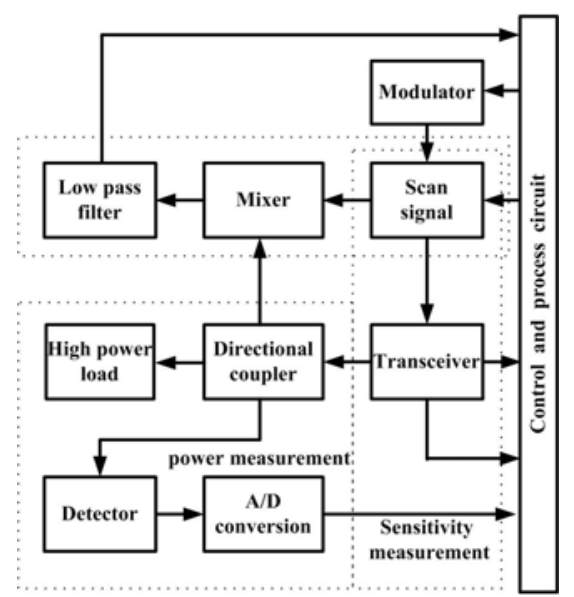

Figure 3. Schematic of microwave signal measurement circuit

\section{b) Frequency-measurement circuit}

The radar-launching signals generated by radar transceiver extension must be within the set range. Otherwise, it will influence the directional pattern of radar antennae, making its main direction deviate from the direction perpendicular to the antennae, then reducing proceeds, weakening the fight function of radar as well. Frequencymeasurement circuit is mainly constituted by sweepingfrequency signal source, mixer (converter) and low-pass filter. Sweep frequency signal source can shift in the range of $100 \mathrm{MHz}$ up and down of center frequency, $1 \mathrm{MHz}$ per step. Signals sent by signal source can make frequency mixing with radar-launching signals sent by directional coupler, then beat frequency will be sent to low-pass filter. The cut-off frequency of low pass filter is $1 \mathrm{MHz}$. That is to say, when the rate of beat frequency is less than $1 \mathrm{MHz}$, low-pass filter has the maximum output, and signal frequency sent by sweeping- frequency signal source is thought to be the frequency of radar-launching signals at this moment, the margin for error of which is not more than $1 \mathrm{MHz}$.Taking out of control voltage output from control circuit, which has a one-to-one correlation with oscillation frequency of sweeping-frequency signal source. Then the measured rate figure will be shown on the monitor.

\section{c) power measurement circuit}

The power of radar transmitter can be expressed by average power and peak power, which directly determines the range that radar works. And the test bench achieves its measurement of radar power through the detector. It is equipped with the average power detector and peak power detector respectively in the test bench. The average power 
detector uses thermistor while the peak power detector uses detector diode. The voltage signal output by detector converts into digital signals by A/D converter and it reads the corresponding power value and displays on the monitor in controlling and processing circuit.

\section{d) Receiver sensitivity measurement circuit}

The sensitivity of Radar receiver uses the least detectable signal to signify , which has a great impact on the radar operating distance. The synchronizing signal of the radar is derived by the radar transceiver extension, and this extension proceeds time delay and pulse width adjustment in controlling and processing circuit. The signal is sent to the modulator to get enlarged, which is used to modulate the sweep frequency signal source. At this moment, the work of sweep frequency signal source outputs the frequency signal modulated by the radar center in a frequency state and sends to the radar indicator through the transceiver extension. Microwave sweep frequency signal source automatically calibrates for $1 \mathrm{~mW}$ by controlling and processing the circuit. The attenuation adopts the digital control way, attenuating with a $10 \mathrm{~dB}$ or $1 \mathrm{~dB}$ for stepping. Microwave sweep frequency signal source comparing the brightness of simulation target signal and the noise on the radar display, and the attenuation volume of the signal is read out by the radar indicator-- radar receiver sensitivity. Compared with the traditional manual decay, the usage of numerical control type decay improves the measuring precision.

\section{DESIGN OF SOFTWARE}

Software of this test bench adopts modular structure, which is initialized by the main program and then inquired by the keyboard to see if there are any buttons to being pressed under the sub procedure. And it calls the corresponding sub procedure according to the menu organizational structure, so that it can complete data acquisition and control function. The platform of the software development uses the integrated environment of Weifu WAVE3.2. The complier uses the C complier of ICES. Structure of the test software is shown in Fig.4.

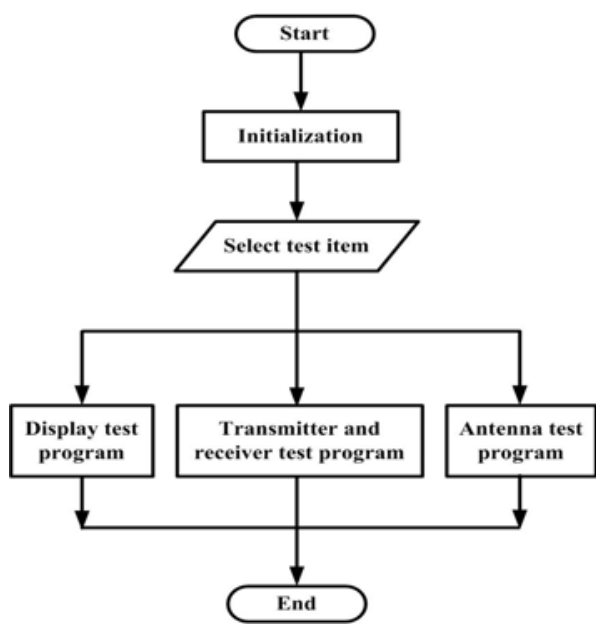

Figure 4. Chart of test software
Because of the Chinese LCD display in the system, we adopt the approach of menu selection as the man-machine information exchange. After starting up , the self-check of the system will be first underwent. This mainly checks whether the working voltage provided for radar is normal or not. If it is in the normal menu option, then it will select the project that needs to be tested. Each test project calls related sub procedure to complete data acquisition and control function.

\section{CONCLUSION}

The designed automatic test bench has achieved all the functions of a certain type of airborne radar testing requirements, which can measure the micro parameters of the radar frequency, power, sensitivity, etc., as well as 27 low-frequency signal parameters, if adapted with various parts of the radar ${ }^{[5]}$. It has solved problems of the differences in the extension interface to be tested, various ranges of the signal to be tested and microwave signal test, as well as many other puzzles. It has achieved resource sharing and automatic test.

This test bench has undergone trails in several corporations since its completion of development in December 2008. It has made automatic test on more than 20 radar equipment, and turned $100 \%$ accuracy in test results. This test bench is in good working condition, with precise test results as well. It also has the advantages of high degree of intelligence, simple operation, reducing human error of maintenance and inspection etc., which has provided effective protection of the maintenance, fault detection and diagnosis in the use of radar. It is prosperous in promotion of application, and has profitable economic benefits.

After the text edit has been completed, the paper is ready for the template. Duplicate the template file by using the Save As command, and use the naming convention prescribed by your conference for the name of your paper. In this newly created file, highlight all of the contents and import your prepared text file. You are now ready to style your paper; use the scroll down window on the left of the MS Word Formatting toolbar.

\section{REFERENCES}

[1] Zeng Xianlin, Li Fuqun, Zhang Kun. A certain Airborne Intelligent Integrated Test System [J]. Computer Measurement\&control, 2008.16 (5) :688-690.

[2] Wen Ruiheng, Ye Wei. Frequency Agile Radar Automated Test and Key Technologies [J]. Computer Measurement\&control, 2009.17 (3) :515-517.

[3] Huang Hexin, Zhu Yongbao. Study of Radar Transmitter Automatic Test System[J]. Computer Measurement\&control, 2000.8 (4) :5859.

[4] Li Jianzhong, Microcontroller Theory and Applications [M]. Xi'an: Xi'an University of Electronic Science and Technology Publishing House, 2008.

[5] Ding Lufei, Geng Fulu. Radar theory [M]. Xi'an: Xi'an University of Electronic Science and Technology Publishing House, 2001. 